\title{
Stripe-tetragonal first-order phase transition in ultrathin magnetic films
}

\author{
Sergio A. Cannas, ${ }^{1, *}$ Daniel A. Stariolo, ${ }^{2, \dagger}$ and Francisco A. Tamarit ${ }^{1, \pitchfork}$ \\ ${ }^{1}$ Facultad de Matemática, Astronomía y Física, Universidad Nacional de Córdoba, Ciudad Universitaria, 5000 Córdoba, Argentina \\ ${ }^{2}$ Departamento de Física, Universidade Federal do Rio Grande do Sul, CP 15051, 91501-979 Porto Alegre, Brazil
}

(Received 20 October 2003; revised manuscript received 2 December 2003; published 30 March 2004)

\begin{abstract}
We analyze the nature of the phase transition from a smectic stripe phase to a tetragonal phase predicted in analytic studies by Abanov et al. [Phys. Rev. B 51, 1023 (1995)] and observed in experiments on ultrathin magnetic films by Vaterlaus et al. [Phys. Rev. Lett. 84, 2247 (2000)]. At variance with existent numerical evidence, we show results of Monte Carlo simulations on a two-dimensional model with competing exchange and dipolar interactions showing strong evidence that the transition is a weak first-order one, in agreement with the theoretical predictions of Abanov et al. Besides the numerical evidence, we give further support to the first-order nature of the transition analyzing a continuum version of the model and showing that it belongs to a large family of systems, or universality class, in which a first-order transition driven by fluctuations is expected on quite general grounds.
\end{abstract}

DOI: 10.1103/PhysRevB.69.092409

With the advances in the experimental manipulation of materials at atomic length scales a renewed interest has grown in understanding the thermodynamic and mechanical properties of systems such as ultrathin films and quasi-twodimensional magnetic materials. Part of this interest is obviously motivated by the great amount of potential applications they find nowadays in many different technological fields (data storage, catalysis, and electronics are only a few examples). In this paper we are mainly concerned with the thermodynamic properties of ultrathin magnetic films, such as metal films on metal substrates (e.g., $\mathrm{Fe}$ on $\mathrm{Cu},{ }^{1} \mathrm{Co}$ on $\mathrm{Au},{ }^{2}$ see also Ref. 3 for a recent review on the topic).

A large variety of magnetic films exhibit a spin reorientation transition below some finite temperature $T_{R}$. That is, if the magnetic film is thin enough (a few atomic layers) the atomic magnetic moments tend to align in a direction perpendicular to the plane of the film, because the surface anisotropy overcomes the anisotropy of the dipolar interactions, which favors in-plane ordering. Under these circumstances the local magnetic moments can be regarded approximately as Ising variables. Any realistic theoretical description of a magnetic thin film must include long-range dipolar interactions. The competition between exchange and dipolar interactions in these materials gives rise to stable modulated stripelike patterns at low temperatures. In these states the magnetic moments align along a particular axis forming ferromagnetic stripes of constant width $h$, so that moments in adjacent stripes are antialigned. Theoretical studies concluded that the stripe state in this system is always the most stable one at low enough temperatures, provided that the exchange parameter exceeds some small positive critical value. ${ }^{4,5}$ Calculations based on a continuum approximation by Abanov et al. ${ }^{4}$ predicted that, before reaching the paramagnetic state at high temperatures, the stripe phase undergoes a transition into a phase characterized by domains with predominantly square corners, which they called a tetragonal liquid. They also concluded that the stripe-tetragonal liquid transition should be either first order or the two phases might be separated by a third phase characterized by rotational domain-wall defects, which they called an Ising nematic phase. Monte Carlo calculations on the square lattice carried
PACS number(s): 75.70.Kw, 75.40.Cx, 75.40.Mg

out by Booth et al. ${ }^{6}$ confirmed the presence of the tetragonal liquid phase, but they did not find any evidence of the Ising nematic phase. However, the results of Booth et al. appeared to be consistent with a continuous transition rather than a first-order one, as could be expected from the theoretical results of Abanov et al. Nevertheless, Booth et al. pointed out that the possibility of a weak first-order transition cannot be excluded on the base of their Monte Carlo simulations. Recent imaging studies using scanning electron microscopy with polarization analysis on ultrathin films of fcc Fe on $\mathrm{Cu}(100)$ verified the existence of the tetragonal liquid phase. ${ }^{7,8}$ No evidence was found of an intermediate nematic phase of the type predicted by Abanov et al. ${ }^{4}$ in the transition from the stripe phase to the tetragonal liquid. The thermodynamic nature of the stripe-tetragonal transition could not be determined by the imaging technique and this question remains unanswered. In this paper we present both Monte Carlo (MC) and analytical calculations that provide evidence that the stripe-tetragonal liquid transition is indeed a weak fluctuation-induced first-order one.

We consider a system of magnetic dipoles on a square lattice in which the magnetic moments are oriented perpendicular to the plane of the lattice, with both nearest-neighbor ferromagnetic exchange interactions and long-range dipoledipole interactions between moments. The thermodynamics of this system is ruled by the dimensionless Hamiltonian: ${ }^{9}$

$$
\mathcal{H}=-\delta \sum_{\langle i, j\rangle} \sigma_{i} \sigma_{j}+\sum_{(i, j)} \frac{\sigma_{i} \sigma_{j}}{r_{i j}^{3}},
$$

where $\delta$ stands for the ratio between the exchange $J_{0}>0$ and the dipolar $J_{d}>0$ interaction parameters, i.e., $\delta=J_{0} / J_{d}$. The first sum runs over all pairs of nearest-neighbor spins and the second one over all distinct pairs of spins of the lattice; $r_{i j}$ is the distance, measured in crystal units, between sites $i$ and $j$. The energy is measured in units of $J_{d}$. The overall (known) features of the equilibrium phase diagram of this system can be found in Refs. 3, 5, 6, and 10, while several dynamical properties at low temperatures can be found in Refs. 11-14. The threshold for the appearance of the stripe phase in this model is $\delta_{c}=0.425 .^{5,9}$ 


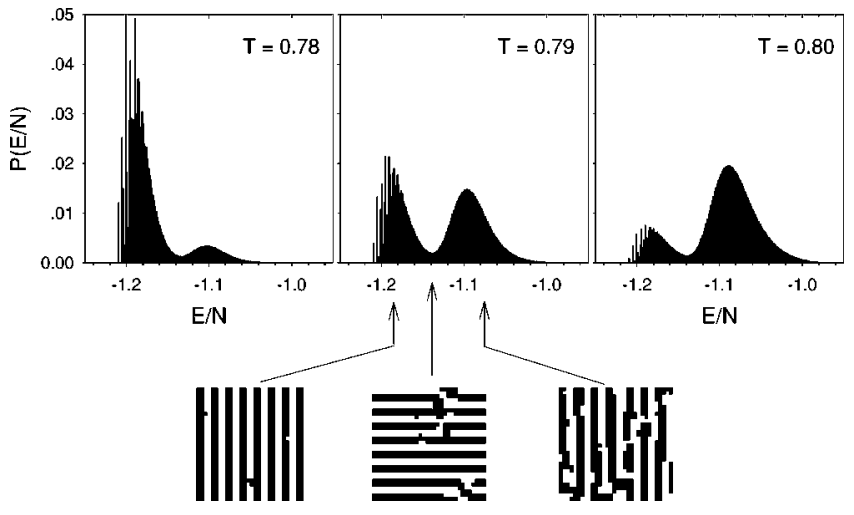

FIG. 1. Energy per spin histograms for $\delta=2, L=32$ and different temperatures around the critical one $T_{c} \approx 0.79$. The images below the histogram illustrate some typical equilibrium spin configurations corresponding to the maxima and the minimum at the critical temperature.

We performed extensive MC simulations of the model defined by Eq. (1) on a square lattice with $N=L^{2}$ sites, for system sizes ranging from $L=16$ to 32 using the Metropolis algorithm. Periodic boundary conditions were implemented using the Ewald summation technique. Although we obtained results for different values of $\delta$ ranging from 1 to 3 , most of the numerical work was focused on $\delta=2$ (corresponding to a low-temperature stripe phase of width $h=2$ ), for which the first-order nature of the transition is more clearly defined. To estimate both equilibration and decorrelation times we analyzed the behavior of the two-times correlation function for different system sizes and temperatures. After an equilibration period of up to $10^{5}$ Monte Carlo steps (MCS), every data point was calculated over a large single run for periods ranging from $2 \times 10^{6} \mathrm{MCS}$ for the smallest size up to $4 \times 10^{7} \mathrm{MCS}$ for the largest one $(L=32)$. To locate the transition and characterize its order we calculated the energy per spin histograms for different temperatures, from which we obtained both the specific heat $C_{L}$ $=\left(1 / N T^{2}\right)\left(\left\langle H^{2}\right\rangle-\langle H\rangle^{2}\right)$ and the fourth-order Binder cumulant ${ }^{15} V_{L}=1-\left\langle H^{4}\right\rangle / 3\left\langle H^{2}\right\rangle^{2}$. The typical behavior of the energy histograms is shown in Fig. 1 for $\delta=2$ and $L$ $=32$. The double-peak structure is characteristic of a first- order phase transition. ${ }^{15,16}$ We observed that the measurement time (in MCS) needed to sample the energy distribution effectively increases exponentially with the linear system size $L$. This is because the system spends large periods of time in each one of the two phases, with a mean lifetime in every phase that increases with $L$, consistently with the expected $^{16}$ increase in the energy-free barrier between phases $\Delta F(L) \sim L^{d-1}$. A careful inspection of the typical equilibrium spin configurations associated with every phase (i.e., those with energies around the maxima of the energy distribution) shows indeed that the high-temperature phase presents a tetragonal structure similar to that found by Booth et $a l .{ }^{6}$ (see Fig. 1), while the low-temperature phase is the $h=2$ stripe one (also notice the coexistence of domains of both phases at energies corresponding to the minimum of the histogram). Moreover, a MC calculation of the structure factor $S(\mathbf{k})=\left\langle\left|\sum_{i} \sigma_{i} e^{i \mathbf{k} \cdot \mathbf{r}_{\mathbf{i}}}\right|^{2}\right\rangle$ at temperatures above, but near the critical one, shows a very similar shape to that observed by MacIsaac et al..$^{5}$ at higher values of $\delta$ : four sharp peaks symmetrically placed on the two principal axes of the Brillouin zone, which characterize the tetragonal structure. ${ }^{3}$ As the temperature increases the peaks become smeared into a circle with the shape of a four-peaked crown that gradually disappears. This indicates a continuous loss of the fourfold symmetry as the system becomes paramagnetic. For large values of $\delta(\delta \geqslant 3)$ this transition is also reflected in the presence of a secondary peak at high temperatures in the specific heat, which does not depend on the system size, ${ }^{6}$ indicating that the transition does not have an associated singularity in the thermodynamic potentials. As already observed by Booth et al., ${ }^{6}$ this secondary peak becomes less pronounced as $\delta$ decreases; we observed that for values of $\delta \leqslant 2.5$ it becomes indistinguishable. However, the presence of the transition remains clearly detectable in the behavior of the structure factor.

The temperature variation of the specific heat and the Binder cumulant for $\delta=2$ and various system sizes is shown in Fig. 2. We see that the locations of the maximum of the specific heat and the minimum of the Binder cumulant shift in a size-dependent fashion at pseudocritical temperatures $T_{c}^{(1)}(L)$ and $T_{c}^{(2)}(L)$, respectively. Both quantities are plotted vs $L^{-2}$ in Fig. 3, showing the expected finite-size scaling
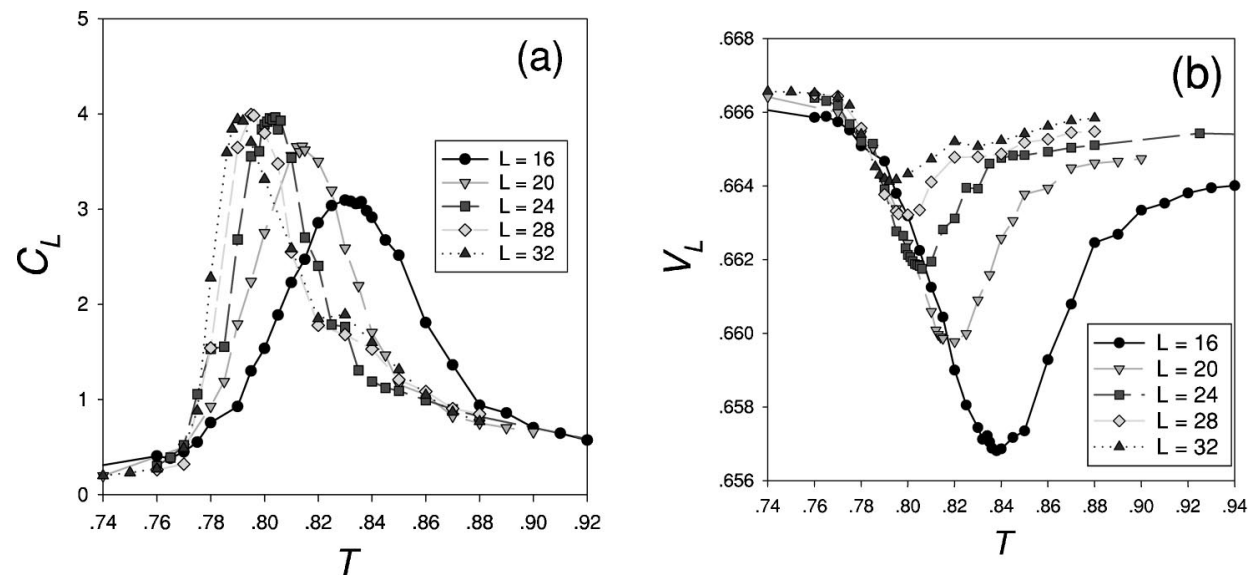

FIG. 2. MC calculations for $\delta=2$ and different system sizes $L$. (a) Specific heat $C_{L}$ vs $T$; (b) Binder cumulant $V_{L}$ vs $T$. 


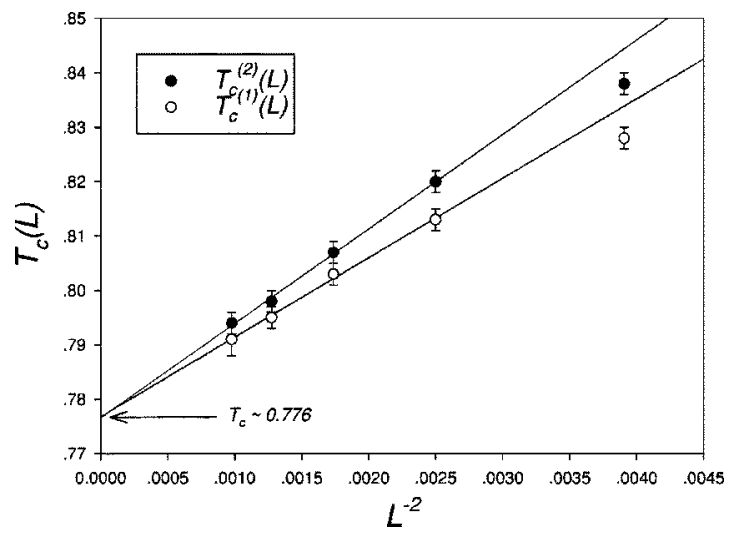

FIG. 3. Pseudocritical temperatures $T_{c}^{(1)}$ (maximum of the specific heat) and $T_{c}^{(2)}$ (minimum of the Binder cumulant) vs $L^{-2}$.

behavior for a temperature-driven first-order phase transition $^{15,16} T_{c}^{(1)}(L) \sim T_{c}+A L^{-d}$ and $T_{c}^{(2)}(L) \sim T_{c}+B L^{-d}$ with $B>A$, where $T_{c}$ is the transition temperature of the infinite system. Note that the internal energies of both phases (corresponding approximately to the energies of the maxima of the energy distribution) are located very close to each other. This property is also reflected in the rather shallow shape of the minimum of the Binder cumulant, evidencing the weak nature of the transition. We observed that these effects become more pronounced as $\delta$ increases, with the internal energies of both phases approaching continuously to each other. For values of $\delta>2.6$ the double-peak structure of the energy histogram (together with the minimum of the Binder cumulant) seems to disappear, or at least it becomes undetectable for small system sizes, as can be appreciated in Fig. 4 for $\delta=2.6$. We see that the internal energies of both phases (roughly corresponding to the energies of the maxima of the histogram) near the transition approach each other as $\delta$ increases. This fact explains the seemingly continuous nature of the transition observed by Booth et al., whose calculations were performed for $\delta \geqslant 3 .{ }^{6}$ Clearly, considerably larger systems must be simulated in order to get reliable data for larger $\delta$. A similar effect is observed as $\delta$ decreases approaching $\delta_{c}$, where the double-peak structure of the histogram also seems to disappear totally. Hence, the numerical data show an optimal value of $\delta$ around $\delta=2$, where the first-order transition becomes strongest, that is, with the largest latent heat. Since strong finite-size effects are always expected in systems with long-range interactions, numerical simulations in considerably larger systems are also required in this case to get a more clear picture about this phenomenon. However, it is worth mentioning that some numerical simulations for larger system sizes ( $L=48$ and $L=64$ ) showed that the double-peak structure of the energy histogram persists and becomes more pronounced as $L$ increases. We now turn our attention to an analytic approximation which gives us some insight in the expected outcome of such simulations for the other regions of the phase diagram.

It was recognized long ago ${ }^{17,18}$ that systems in which the spectrum of fluctuations has a minimum at a nonzero wave vector can undergo a first-order transition driven by fluctuations, in contrast to the second-order transition predicted by mean field for this kind of systems. Since the original work by Brazovskii, the proposed scenario was shown to describe correctly the phase transitions present in a large variety of systems such as cholesteric liquid crystals, the nematic to smectic- $C$ transition, pion condensates in neutron stars, onset of Rayleigh-Bénard convection, and microphase separation in diblock copolymers. ${ }^{18,19}$ More recently the Brazovskii scenario has been successfully applied to the analysis of the phase transition between the disordered and modulated phases in three-dimensional systems with attractive shortrange interactions and repulsive long-range Coulomb interactions. $^{20,21}$ In spite of its success and ubiquity in correctly describing the physics behind a phase transition in systems with competing interactions, the Brazovskii scenario was almost not considered in relation with ultrathin films and dipolar systems. ${ }^{22}$ Indeed we have verified that a continuum version of Hamiltonian (1) presents a fluctuation induced first-order phase transition for any value of $\delta$. The starting point is a Landau-Ginzburg functional which in Fourier space has the form

$$
\begin{aligned}
\mathcal{H}= & \frac{V}{2} \int \frac{d \vec{k}}{(2 \pi)^{2}} A(k) \phi(\vec{k}) \phi(-\vec{k})+\frac{u V}{4} \int \frac{d \vec{k}_{1}}{(2 \pi)^{2}} \frac{d \vec{k}_{2}}{(2 \pi)^{2}} \\
& \times \frac{d \vec{k}_{3}}{(2 \pi)^{2}} \phi\left(\vec{k}_{1}\right) \phi\left(\vec{k}_{2}\right) \phi\left(\vec{k}_{3}\right) \phi\left(-\vec{k}_{1}-\vec{k}_{2}-\vec{k}_{3}\right)
\end{aligned}
$$

where the spectrum of fluctuations is given by $A(k)=r_{0}$ $+k^{2}+J(k) / \delta$ and $r_{0}$ is proportional to the reduced temperature near the critical point of the mean-field approxima-
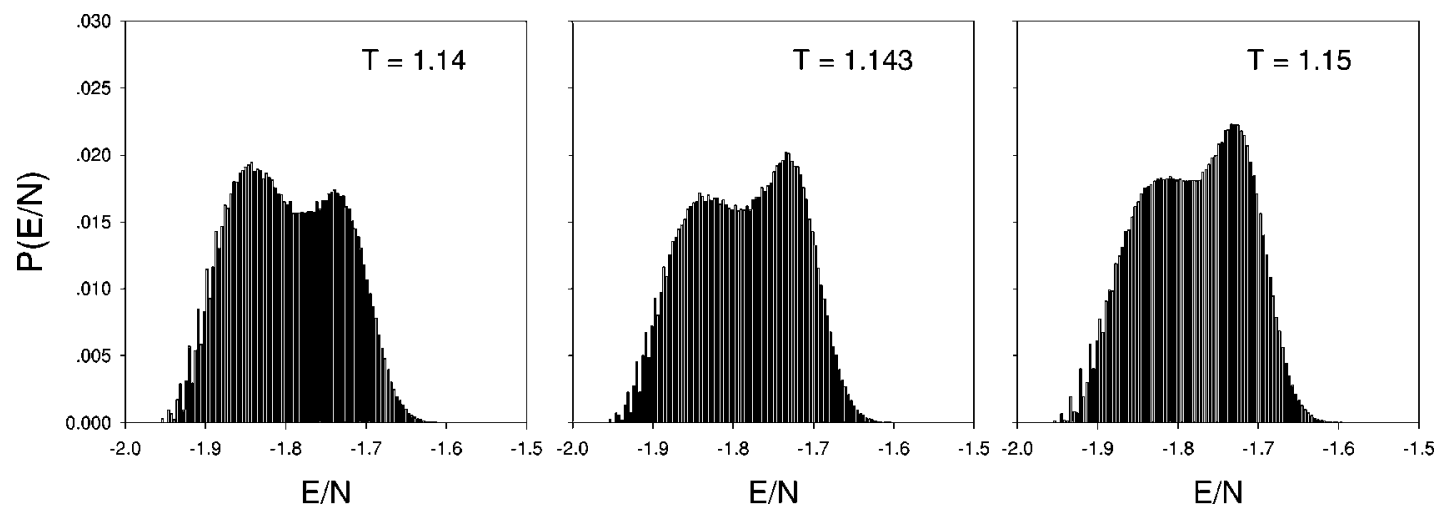

FIG. 4. Energy per spin histograms for $\delta=2.6, L=32$ and different temperatures around the critical one. 
tion. In our case the Fourier transform of the dipolar interacis $J(k)={ }_{1} F_{2}\left(\{-1 / 2\},\{1 / 2,1\},-k^{2} / 4\right)-k$, where ${ }_{1} F_{2}(\{\alpha\}$, $\left.\left\{\beta_{1}, \beta_{2}\right\}, z\right)$ is a generalized hypergeometric series. ${ }^{23}$ The spectrum of fluctuations presents a minimum at a nonzero $k_{\min }=k_{0}$. This means that, for $d \geqslant 2$, the spectrum is minimized in a spherical shell in Fourier space. When fluctuations of the order parameter are considered self-consistently, the degeneracy of the minimizing vector will make the disordered phase metastable for any finite temperature and drive the transition to first order. More explicitly, within a Hartree approximation the correlation function of the fluctuations in the disordered phase is given by

$$
C^{-1}(\vec{k})=A(k)+3 u \int \frac{d \vec{q}}{(2 \pi)^{2}} C(\vec{q}) .
$$

Then, the renormalized mass $r=r_{0}+3 u \int\left[d \vec{q} /(2 \pi)^{2}\right] C(\vec{q})$ is given by

$$
r=r_{0}+3 u \int \frac{d \vec{q}}{(2 \pi)^{2}} \frac{1}{r+q^{2}+J(q) / \delta} .
$$

The point of absolute instability of the disordered solution occurs at $r=-k_{0}^{2}-J\left(k_{0}\right) / \delta$. It is easy to check that this point is only reached for $r_{0} \rightarrow-\infty$ (i.e., for $T \rightarrow 0$ ), implying that the disordered phase never loses stability. At a finite temperature a modulated phase with a finite amplitude given by $m^{2}=\left[r_{m}+k_{0}^{2}+J\left(k_{0}\right) / \delta\right] / 3 u$ appears, where the renormalized mass in the modulated phase $r_{m}$ is given selfconsistently by

$$
-r_{m}=r_{0}+3 u \int \frac{d \vec{q}}{(2 \pi)^{2}} \frac{1}{r_{m}+q^{2}+J(q) / \delta}+2\left(k_{0}^{2}+\frac{J\left(k_{0}\right)}{\delta}\right) .
$$

In a region $r_{0} \leqslant r_{0}^{*}(\delta)$ a real solution to the above equation exists. If a point $r_{0}^{c}<r_{0}^{*}$ exists where the free energies of the modulated and disordered solutions cross each other, then a first-order transition driven by fluctuations appears. Following Brazovskii ${ }^{17}$ we have determined the point $r_{0}^{c}(\delta)$ where the free-energy difference

$$
\begin{aligned}
u \Delta F= & \int_{r}^{r_{m}} d r^{\prime}\left(\frac{1}{6}+\frac{u}{2} \int \frac{d \vec{q}}{(2 \pi)^{2}} \frac{1}{\left(r^{\prime}+q^{2}+J(q) / \delta\right)^{2}}\right) \\
& \times\left(\frac{r^{\prime}+r_{0}}{2}+k_{0}^{2}+\frac{J\left(k_{0}\right)}{\delta}\right. \\
& \left.+\frac{3 u}{2} \int \frac{d \vec{q}}{(2 \pi)^{2}} \frac{1}{r^{\prime}+q^{2}+J(q) / \delta}\right)
\end{aligned}
$$

changes sign. It is possible to show that for any $\delta>0$ a first-order transition appears. The transition line $r_{0}^{c}(\delta)$ is a monotonously increasing function of $\delta$.

In summary, we have shown strong numerical evidence that the phase transition between the low-temperature stripe phase and the tetragonal phase observed in ultrathin magnetic films is a weak first-order transition. Moreover, to the extent to which a continuum Hartree approximation of the model simulated is valid, the transition is a first-order one driven by fluctuations, at variance with the second-order nature predicted by mean-field theory. It would be interesting to perform simulations in larger lattices and with smarter Monte Carlo algorithms in order to analyze if the first-order nature extends to a wider region of the phase diagram as implied by the continuum model. The challenge persists to probe the nature of this transition experimentally. ${ }^{7,8}$

This work was partially supported by grants from Consejo Nacional de Investigaciones Científicas y Técnicas CONICET (Argentina), Agencia Córdoba Ciencia (Córdoba, Argentina), Secretaría de Ciencia y Tecnología de la Universidad Nacional de Córdoba (Argentina), Fundação VITAE (Brazil), and CNPq (Brazil). We wish to thank R. DíazMéndez and Roberto Mulet for their help in the implementation of the Ewald sums.
*Member of CONICET, Argentina. Electronic address:
cannas@ famaf.unc.edu.ar
${ }^{\dagger}$ Also at: Abdus Salam International Centre for Theoretical Physics,
Trieste, Italy. Electronic address: stariolo@if.ufrgs.br

${ }^{\ddagger}$ Member of CONICET, Argentina. Electronic address: tamarit@famaf.unc.edu.ar

${ }^{1}$ D.P. Pappas et al., Phys. Rev. Lett. 64, 3179 (1990).

${ }^{2}$ R. Allenspach, et al., Phys. Rev. Lett. 65, 3344 (1990).

${ }^{3}$ K. De'Bell, et al., Rev. Mod. Phys. 72, 225 (2000).

${ }^{4}$ A. Abanov, et al., Phys. Rev. B 51, 1023 (1995).

${ }^{5}$ A.B. MacIsaac et al., Phys. Rev. B 51, 16033 (1995).

${ }^{6}$ I. Booth et al., Phys. Rev. Lett. 75, 950 (1995).

${ }^{7}$ A. Vaterlaus et al., Phys. Rev. Lett. 84, 2247 (2000).

${ }^{8}$ O. Portmann, et al., Nature (London) 422, 701 (2003).

${ }^{9}$ It is worth noting that the definition of the Hamiltonian in Refs. 3,5 , and 6 is slightly different from ours. While in those papers the dipolar term contains a sum over all pairs of spins, here we consider the sum over every pair of spins just once. This leads to the equivalence $\delta=J / 2, J$ being the exchange parameter in the above references. Since the dipolar parameter also fixes the energy units in our work, there is also a factor $1 / 2$ between the critical temperatures obtained in those works and ours.

${ }^{10}$ P.M. Gleiser, et al., Physica D 168-169, 73 (2002).

${ }^{11}$ M.L.C. Sampaio, Phys. Rev. B 54, 6465 (1996).

${ }^{12}$ J.H. Toloza, et al., Phys. Rev. B 58, R8885 (1998).

${ }^{13}$ D.A. Stariolo and S.A. Cannas, Phys. Rev. B 60, 3013 (1999).

${ }^{14}$ P.M. Gleiser et al., Phys. Rev. B 68, 134401 (2003).

${ }^{15}$ M.S.S. Challa, et al., Phys. Rev. B 34, 1841 (1986).

${ }^{16}$ J. Lee and J.M. Kosterlitz, Phys. Rev. B 43, 3265 (1991).

${ }^{17}$ S.A. Brazovskii, Sov. Phys. JETP 41, 85 (1975).

${ }^{18}$ P.C. Hohenberg and J.B. Swift, Phys. Rev. E 52, 1828 (1995).

${ }^{19}$ M. Seul and D. Andelman, Science 267, 476 (1995).

${ }^{20}$ M. Grousson, et al., Phys. Rev. E 66, 026126 (2002).

${ }^{21}$ P. Viot and G. Tarjus, Europhys. Lett. 44, 423 (1998).

${ }^{22}$ T. Garel and S. Doniach, Phys. Rev. B 26, 325 (1982).

${ }^{23}$ I. S. Gradshteyn and I. M. Ryzhik, Table of Integrals, Series and Products, 5th ed. (Academic Press, New York, 1994). 